\title{
A COUNTEREXAMPLE IN NONLINEAR INTERPOLATION ${ }^{1}$
}

\author{
MICHAEL CWIKEL
}

\begin{abstract}
If $\left(A_{0}, A_{1}\right)$ is an interpolation pair with $A_{0} \subset A_{1}$ and $T$ is a possibly nonlinear operator which maps $A_{0}$ into $A_{0}$ and $A_{1}$ into $A_{1}$ and satisfies $\|T a\|_{A_{0}} \leqslant C\|a\|_{A_{0}}$ and $\left\|T b-T b^{\prime}\right\|_{A_{1}} \leqslant C\left\|b-b^{\prime}\right\|_{A_{1}}$ for all $a \in A_{0}$ and $b, b^{\prime} \in A_{1}$ and for some constant $C$, then it is known that $T$ also maps the real interpolation spaces $\left(A_{0}, A_{1}\right)_{\theta, p}$ into themselves. We give an example showing that $T$ need not map the complex interpolation spaces $\left[A_{0}, A_{1}\right]_{0}$ into themselves. It is also seen that quasilinear operators may fail to preserve complex interpolation spaces.
\end{abstract}

Introduction. The theory of interpolation spaces, which was originally developed as a generalization of classical results concerning interpolation of linear operators, also provides a setting for results concerning nonlinear operators. The following is a rudimentary example of a theorem for "Lipschitz" operators.

THEOREM 1. Let $\left(A_{0}, A_{1}\right)$ be an interpolation pair with $A_{0} \subset A_{1}$ and let $T$ be a possibly nonlinear operator mapping $A_{0}$ into $A_{0}$ and $A_{1}$ into $A_{1}$ such that

(i) $\|T a\|_{A_{0}} \leqslant C\|a\|_{A_{0}}$,

(ii) $\left\|T b-T b^{\prime}\right\|_{A_{1}} \leqslant C\left\|b-b^{\prime}\right\|_{A_{1}}$

for all $a \in A_{0}, b, b^{\prime} \in A_{1}$ and some constant $C$. Then $T$ maps $\left(A_{0}, A_{1}\right)_{\theta, p}$ into itself for all $\theta, 0<\theta<1$ and all $p, 0<p \leqslant \infty$.

The proof is immediate and amounts to showing that $K\left(t, T f ; A_{0}, A_{1}\right)$ $\leqslant C K\left(t, f ; A_{0}, A_{1}\right)$ where $K$ is the Peetre functional [3]. We refer for example to [4], [5], and [7] for variants, generalizations, and applications of this theorem.

The purpose of this note is to provide a solution to the problem, posed by J.-L. Lions, of finding an example of an operator $T$ which satisfies the above hypotheses but does not map the complex interpolation spaces $\left[A_{0}, A_{1}\right]_{\theta}$ into themselves. As remarked by Professor Lions, it is hardly to be expected that such a nonlinear operator would preserve complex interpolation spaces. But in trying to show this in practice one is faced by the problem that for many important and well-studied interpolation pairs the complex interpolation

Received by the editors December 17, 1975 and, in revised form, March 30, 1976.

AMS (MOS) subject classifications (1970). Primary 46E35; Secondary 46E30.

Key words and phrases. Interpolation of nonlinear operators.

${ }^{1}$ Research supported by the C.N.R.S. 
spaces coincide with suitably chosen real interpolation spaces and so a counterexample must be sought using other pairs where this coincidence does not occur. It seems that the first case where it was observed that real and complex interpolation spaces do not coincide was for the pair $\left(L^{p}, W^{1, p}\right)$ on the circle. This follows from an argument using lacunary series due (independently) to J.-P. Kahane and E. M. Stein (see e.g. [6, Chapter IX]). However, by much simpler arguments we can see that the real and complex interpolation spaces for the pair $\left(l^{1} \oplus l^{2}, l^{\infty} \oplus l^{\infty}\right)$ also fail to coincide, and for our purposes it is apparently rather easier to use this pair.

Construction of the operator. We shall use the spaces $L^{p}, 1 \leqslant p \leqslant \infty$, and $L^{p, q}, 1 \leqslant p, q<\infty$, defined with respect to Lebesgue measure on the positive real line (see [2]). We shall also need the analogous spaces $l^{p}, l^{p, q}$ of sequences on the positive integers. We recall that for $1 \leqslant p<\infty, 0<\theta<1$ and 1 $\leqslant q \leqslant \infty$

$$
\left(L^{p}, L^{\infty}\right)_{\theta, q}=L^{p /(1-\theta), q},\left(l^{p}, l^{\infty}\right)_{\theta, q}=l^{p /(1-\theta), q},
$$

and $K\left(t, f ; L^{p}, L^{\infty}\right)$ is equivalent to $\left(\int_{0}^{t^{p}}\left(f^{*}(s)\right)^{p} d s\right)^{1 / p}$, where $f^{*}(t)$ is the nonincreasing rearrangement of the function $f(t)[3]$. Also $\left[L^{p}, L^{\infty}\right]_{\theta}=L^{p /(1-\theta)}$ and $\left[l^{p}, l^{\infty}\right]_{\theta}=l^{p /(1-\theta)}[1]$.

We begin by constructing an operator $S$ which is a bounded map of $L^{2}$ into $L^{1}$ and of $L^{\infty}$ into $L^{\infty} . S$ is chosen to satisfy

$$
K\left(t, S f ; L^{1}, L^{\infty}\right) \approx K\left(t, f ; L^{2}, L^{\infty}\right)
$$

and in fact is defined, roughly speaking, by differentiating this equation. It is seen that $S$ is Lipschitz on $L^{\infty}$ (and also quasilinear). Picking $g$ $\in L^{1 /(1-\theta), 2 /(1-\theta)} \backslash L^{1 /(1-\theta)}$ and setting $h=S^{-1} g$, we see that, by (*),

$$
\|h\|_{L^{2 /(1-\theta), 2 /(1-\theta)}} \approx\|S h\|_{L^{1 /(1-\theta), 2 /(1-\theta)}}=\|g\|_{L^{1 /(1-\theta), 2 /(1-\theta)}}<\infty
$$

that is, $h$ is in $L^{2 /(1-\theta)}$ but $S h=g$ is not in $L^{1 /(1-\theta)}$ and $S$ does not preserve complex interpolation spaces. By discretising $S$ to an operator $T$ on sequence spaces and then reinterpreting $T$ as an operator on direct sums of sequence spaces, we obtain the required form of the counterexample.

The operator $S$ which acts on $L^{2}+L^{\infty}$ will be defined by:

$$
\begin{aligned}
& S f(t)=0 \text { if } f \text { is identically zero, } \\
& S f(t)=\left(f^{*}\left(t^{2}\right)\right)^{2}\left(t^{-2} \int_{0}^{t^{2}}\left(f^{*}(s)\right)^{2} d s\right)^{-1 / 2} \text { otherwise. }
\end{aligned}
$$

LEMMA 1. $S$ maps $L^{2}$ into $L^{1}$ and $L^{\infty}$ into $L^{\infty}$ such that

(i) $\|S f\|_{L^{1}}=\|f\|_{L^{2}}$ for all $f \in L^{2}$,

(ii) $\|S f\|_{L^{\infty}} \leqslant\|f\|_{L^{\infty}}$ for all $f \in L^{\infty}$,

(iii) $\|S f-S g\|_{L^{\infty}} \leqslant 3\|f-g\|_{L^{\infty}}$ for all $f, g \in L^{\infty}$. 


\section{Furthermore,}

(iv) $K\left(t, S f ; L^{1}, L^{\infty}\right) \geqslant C K\left(t, f ; L^{2}, L^{\infty}\right)$ for all positive $t$, all $f \in L^{2}+L^{\infty}$ and some constant $C>0$.

Proof. $S f(t)=(d / d t)\left(\int_{0}^{t^{2}}\left(f^{*}(s)\right)^{2} d s\right)^{1 / 2}$ for almost all positive $t$. Since $\left(\int_{0}^{t^{2}}\left(f^{*}(s)\right)^{2} d s\right)^{1 / 2}$ is an absolutely continuous function of $t$ on every compact subinterval of $(0, \infty), \int_{0}^{t} S f(s) d s=\left(\int_{0}^{t^{2}}\left(f^{*}(s)\right)^{2} d s\right)^{1 / 2}$. Letting $t$ tend to infinity we have (i). Also $\int_{0}^{t}(S f)^{*}(s) d s \geqslant\left(\int_{0}^{t^{2}}\left(f^{*}(s)\right)^{2} d s\right)^{1 / 2}$ which proves (iv). The fact that $f^{*}$ is nonincreasing immediately implies (ii).

It remains to prove (iii). Suppose that $f_{0}(t)$ and $f_{1}(t)$ are bounded functions with nonincreasing rearrangements $f_{0}^{*}(t)$ and $f_{1}^{*}(t)$, and that neither of them is identically zero. First note that $\left|f_{0}(s)\right| \leqslant\left\|f_{0}-f_{1}\right\|_{L^{\infty}}+\left|f_{1}(s)\right|$ and, consequently, $f_{0}^{*}(t) \leqslant\left\|f_{0}-f_{1}\right\|_{L^{\infty}}+f_{1}^{*}(t)$. It follows that

$$
\left\|f_{0}^{*}-f_{1}^{*}\right\|_{L^{\infty}} \leqslant\left\|f_{0}-f_{1}\right\|_{L^{\infty}} .
$$

Let $E=\left\{t>0 \mid f_{0}^{*}\left(t^{2}\right) \leqslant f_{1}^{*}\left(t^{2}\right)\right\}$ and let $j(t)=\chi_{E}(t)$ and $k(t)=1-j(t)$. Then $f_{j(t)}^{*}\left(t^{2}\right) \geqslant f_{k(t)}^{*}\left(t^{2}\right)$ for all $t>0$.

$$
\begin{aligned}
\mid S f_{0}(t) & -S f_{1}(t)|\leqslant|\left(f_{j(t)}^{*}\left(t^{2}\right)\right)^{2}-\left(f_{k(t)}^{*}\left(t^{2}\right)\right)^{2} \mid\left(t^{-2} \int_{0}^{t^{2}}\left(f_{j(t)}^{*}(s)\right)^{2} d s\right)^{-1 / 2} \\
& +\left(f_{k(t)}^{*}\left(t^{2}\right)\right)^{2}\left|\left(t^{-2} \int_{0}^{t^{2}}\left(f_{j(t)}^{*}(s)\right)^{2} d s\right)^{-1 / 2}-\left(t^{-2} \int_{0}^{t^{2}}\left(f_{k(t)}^{*}(s)\right)^{2} d s\right)^{-1 / 2}\right| .
\end{aligned}
$$

The first term is dominated by $2\left|f_{0}^{*}\left(t^{2}\right)-f_{1}^{*}\left(t^{2}\right)\right| \leqslant 2\left\|f_{0}-f_{1}\right\|_{L^{\infty}}$ and the second term is dominated by

$$
\begin{gathered}
\frac{f_{0}^{*}\left(t^{2}\right) f_{1}^{*}\left(t^{2}\right)\left|\left(t^{-2} \int_{0}^{t^{2}}\left(f_{0}^{*}(s)\right)^{2} d s\right)^{1 / 2}-\left(t^{-2} \int_{0}^{t^{2}}\left(f_{1}^{*}(s)\right)^{2} d s\right)^{1 / 2}\right|}{\left(t^{-2} \int_{0}^{t^{2}}\left(f_{0}^{*}(s)\right)^{2} d s\right)^{1 / 2}\left(t^{-2} \int_{0}^{t^{2}}\left(f_{1}^{*}(s)\right)^{2} d s\right)^{1 / 2}} \\
\leqslant \frac{t^{-2} \int_{0}^{t^{2}}\left|\left(f_{0}^{*}(s)\right)^{2}-\left(f_{1}^{*}(s)\right)^{2}\right| d s}{\left(t^{-2} \int_{0}^{t^{2}}\left(f_{0}^{*}(s)\right)^{2} d s\right)^{1 / 2}+\left(t^{-2} \int_{0}^{t^{2}}\left(f_{1}^{*}(s)\right)^{2} d s\right)^{1 / 2}} \\
\leqslant \frac{\left\|f_{0}-f_{1}\right\|_{L^{\infty}} t^{-2} \int_{0}^{t^{2}} f_{0}^{*}(s)+f_{1}^{*}(s) d s}{\left(t^{-2} \int_{0}^{t^{2}}\left(f_{0}^{*}(s)\right)^{2} d s\right)^{1 / 2}+\left(t^{-2} \int_{0}^{t^{2}}\left(f_{1}^{*}(s)\right)^{2} d s\right)^{1 / 2}} \\
\leqslant\left\|f_{0}-f_{1}\right\|_{L^{\infty}} .
\end{gathered}
$$

(iii) follows and the lemma is proved.

Let the operator $T$ on $L^{2}+L^{\infty}$ be defined by

$$
T f(t)=\int_{[t]}^{[t]+1} S f(s) d s
$$

where $[t]$ is the integer part of $t$. The functions $T f$ are, of course, constant on 
each interval $[n, n+1), n=0,1,2, \ldots$, and we may alternatively consider them as sequences. Furthermore, considering the restriction of $T$ to the subspace $l^{2}+l^{\infty}$ of $L^{2}+L^{\infty}$ which consists of functions constant on each interval $[n, n+1)$, we deduce immediately that $T$ maps $l^{\infty}$ into $l^{\infty}$ and $l^{2}$ into $l^{1}$ with $\|T f\|_{l^{1}}=\|f\|_{l^{2}}$ for all $f \in l^{2}$ and $\|T f-T g\|_{l^{\infty}} \leqslant 3\|f-g\|_{l^{\infty}}$ for all $f$ and $g$ in $l^{\infty}$. By an obvious variant of Theorem $1, T$ maps $\left(l^{2}, l^{\infty}\right)_{\theta, p}$ into $\left(l^{1}, l^{\infty}\right)_{\theta, p}$ for each $\theta \in(0,1)$ and $p \in(0, \infty]$. We shall see that $T$ does not map $\left[l^{2}, l^{\infty}\right]_{\theta}$ into $\left[l^{1}, l^{\infty}\right]_{\theta}$ for any $\theta$. This will prove the conjecture of Lions in a slightly different form. The counterexample which proves the conjecture in the form stated above is obtained using the interpolation pair $\left(l^{2} \oplus l^{1}, l^{\infty} \oplus l^{\infty}\right)$ and the operator $U$ defined by $U(f, g)=(0, T f)$ for each pair $(f, g) \in l^{\infty} \oplus l^{\infty}$.

Let $g(t)$ be a decreasing step function on $(0, \infty)$ with $g(t)=g_{n}$ on $(n-1, n]$, $n=1,2, \ldots$ In particular, if $g_{n}=n^{-(1-\theta)}(\log n+1)^{-3(1-\theta) / 4}$ we have $g$ $\notin l^{1 /(1-\theta)}=\left[l^{1}, l^{\infty}\right]_{\theta}$, but

$$
g \in l^{1 /(1-\theta), 2 /(1-\theta)}=\left(l^{1}, l^{\infty}\right)_{\theta, 2 /(1-\theta)} .
$$

For all $t>0$ let $h(t)=\left(t^{-1 / 2} \int_{0}^{t^{1 / 2}} g(s) d s\right)^{1 / 2}\left(g\left(t^{1 / 2}\right)\right)^{1 / 2}$. Then $h(t)$ must also be a bounded decreasing function, and one can readily verify that $S h(t)$ $=g(t)$. Let $a(t)$ and $b(t)$ be two step functions defined by $a(t)=h\left([\sqrt{ } t]^{2}\right), b(t)$ $=h\left([\sqrt{ } t+1]^{2}\right)$, where again $[\cdot]$ denotes "integer part", and where $h(0)$ is taken to be $\lim _{t \rightarrow 0} h(t)=g_{1}$. Then $b(t) \leqslant h(t) \leqslant a(t)$ and $a(t)$ and $b(t)$ are constant on the intervals $\left[n^{2},(n+1)^{2}\right), n=0,1,2, \ldots$,

$$
\begin{aligned}
\sup _{t>0} \frac{a(t)}{b(t)} & =\sup _{n=0}^{\infty} \frac{h\left(n^{2}\right)}{h\left((n+1)^{2}\right)} \\
& \leqslant \frac{h(0)}{h(1)}+\sup _{n=1}^{\infty}\left(\frac{n+1}{n}\right)^{1 / 2}\left(\frac{g(n)}{g(n+1)}\right)^{1 / 2} \\
& \leqslant M, \text { where } M \text { is a finite positive constant. }
\end{aligned}
$$

Consequently, $h(t) \leqslant a(t) \leqslant M h(t)$ and so $M^{-1} S h(t) \leqslant S a(t) \leqslant M^{2} \operatorname{Sh}(t)$. Considering $a(t)$ as a sequence in $l^{\infty}$ we see that $T a$, like $g$, is not in $\left[l^{1}, l^{\infty}\right]_{\theta}$. However, $a$ is in $\left[l^{2}, l^{\infty}\right]_{\theta}$, since $\left[l^{2}, l^{\infty}\right]_{\theta}=\left(l^{2}, l^{\infty}\right)_{\theta, 2 /(1-\theta)}$ and

$$
\begin{aligned}
K\left(t, a ; l^{2}, l^{\infty}\right) & =K\left(t, a ; L^{2}, L^{\infty}\right) \leqslant \text { const } K\left(t, S a ; L^{1}, L^{\infty}\right) \\
& \leqslant \text { const } K\left(t, g ; L^{1}, L^{\infty}\right)
\end{aligned}
$$

by (iv) of Lemma 1 , and $g \in\left(l^{1}, l^{\infty}\right)_{\theta, 2 /(1-\theta)} \subset\left(L^{1}, L^{\infty}\right)_{\theta, 2 /(1-\theta)}$. Therefore $T$ does not map $\left[l^{2}, l^{\infty}\right]_{\theta}$ into $\left[l^{1}, l^{\infty}\right]_{\theta}$.

REMARK. An operator $Q$ mapping $A_{j}$ into $B_{j}, j=0$, where $\left(A_{0}, A_{1}\right)$, $\left(B_{0}, B_{1}\right)$ are interpolation pairs, is said to be quasilinear (Krée [3]) if there exists a constant $C$ such that

(i) $\|Q a\|_{B_{j}} \leqslant C\|a\|_{A_{j}}$ for all $a \in A_{j}, j=0,1$,

(ii) $Q$ maps $A_{0}+A_{1}$ into $B_{0}+B_{1}$ and for each pair $a_{0} \in A_{0}, a_{1} \in A_{1}$ there 
exist $b_{0} \in B_{0}, b_{1} \in B_{1}$ such that $Q\left(a_{0}+a_{1}\right)=b_{0}+b_{1}$ and $\left\|b_{j}\right\|_{B_{j}} \leqslant C\left\|a_{j}\right\|_{A_{j}}$, $j=0,1$.

It is immediately apparent that such an operator must map $\left(A_{0}, A_{1}\right)_{\theta, p}$ into $\left(B_{0}, B_{1}\right)_{\theta, p}$ for $0<\theta<1,1 \leqslant p \leqslant \infty$, and again one could ask whether $\left[A_{0}, A_{1}\right]_{\theta}$ is mapped to $\left[B_{0}, B_{1}\right]_{\theta}$. Here also the answer is negative. The operator $S$ is a quasilinear operator from $\left(L^{2}, L^{\infty}\right)$ to $\left(L^{1}, L^{\infty}\right)$ but does not map $\left[L^{2}, L^{\infty}\right]_{\theta}$ to $\left[L^{1}, L^{\infty}\right]_{\theta}$. To see this, first note that as a function $g=s h$ $\notin\left[L^{1}, L^{\infty}\right]_{\theta}$ but $h \in\left[L^{2}, L^{\infty}\right]_{\theta}$. Now let $f_{0} \in L^{2}, f_{1} \in L^{\infty}$ and let $f=f_{0}+f_{1}$. Then there exist functions $g_{0}, g_{1}$, with $0 \leqslant g_{j}(t) \leqslant\left|f_{j}(t)\right|, j=0,1$, and $g_{0}(t)$ $+g_{1}(t)=|f(t)|$. So $f^{*}(t) \leqslant g_{0}^{*}(t / 2)+g_{1}^{*}(t / 2)[2$, p. 253] and

$$
\begin{aligned}
S f(t) & \leqslant\left(g_{0}^{*}\left(t^{2} / 2\right)+g_{1}^{*}\left(t^{2} / 2\right)\right)^{2}\left(t^{-2} \int_{0}^{t^{2} / 2}\left(f^{*}(s)\right)^{2} d s\right)^{-1 / 2} \\
& \leqslant 2 \sqrt{2}\left[\left(S g_{0}\right)(t / \sqrt{ } 2)+\left(S g_{1}\right)(t / \sqrt{ } 2)\right] .
\end{aligned}
$$

From this it is clear that $S$ is quasilinear.

\section{REFERENCES}

1. A.-P. Calderón, Intermediate spaces and interpolation, the complex method, Studia Math. 24 (1964), 113-190. MR 29 \#5097.

2. R. A. Hunt, On $L(p, q)$ spaces, Enseignement Math. (2) 12 (1966), 249-276. MR 36 \#6921.

3. P. Krée, Interpolation d'espaces qui ne sont ni normés, ni complets. Applications, Ann. Inst. Fourier (Grenoble) 17 (1967), fasc. 2, 137-174 (1968). MR 37 \#4605.

4. J.-L. Lions, Some remarks on variational inequalities, Proc. Internat. Conf. Functional Analysis and Related Topics (Tokyo, 1969), Univ. of Tokyo Press, Tokyo, 1970, pp. 269-282. MR 42 \#2322.

5. J. Peetre, Interpolation of Lipschitz operators and metric spaces, Math. Cluj. 12 (1970), 325-334.

6. M. H. Taibleson, On the theory of Lipschitz spaces of distributions on Euclidean n-space. I. Principal properties, J. Math. Mech. 13 (1964), 407-479. MR 29 \#462.

7. L. Tartar, Interpolation non linéaire et régularité, J. Functional Analysis 9 (1972), 469-489. MR 46 \#9717.

Universite de Paris-Sud, Centre Scientifique d’Orsay, Mathématiques (Bât. 425), 91405 Orsay, France

Current address: Department of Mathematics, Technion, Israel Institute of Technology, Haifa, Israel 